\title{
HST observations of the pulsating white dwarf GD 358
}

\author{
B. G. Castanheira ${ }^{1}$, A. Nitta ${ }^{2}$, S. O. Kepler ${ }^{1}$, D. E. Winget ${ }^{3}$, and D. Koester ${ }^{4}$ \\ ${ }^{1}$ Instituto de Física, Universidade Federal do Rio Grande do Sul, 91501-900 Porto-Alegre, RS, Brazil \\ e-mail: barbara@if.ufrgs.br \\ 2 Sloan Digital Sky Survey, Apache Pt. Observatory, PO Box 59, Sunspot, NM 88349, USA \\ 3 Department of Astronomy and McDonald Observatory, University of Texas, Austin, TX 78712, USA \\ 4 Institut für Theoretische Physik und Astrophysik, Universität Kiel, 24098 Kiel, Germany
}

Received 2 July 2004 / Accepted 20 October 2004

\begin{abstract}
We used time-resolved ultraviolet spectroscopy obtained with the FOS and STIS spectrographs of the Hubble Space Telescope (HST), together with archival IUE observations to measure the effective temperature ( $T_{\text {eff }}$ ), surface gravity $(\log g)$ and distance $(d)$ of the pulsating DB white dwarf GD 358 with unprecedented accuracy, and to show that the temperature did not change during the 1996 sforzando, when the star changed basically to a single mode pulsator. We also measured for the first time for a DBV the spherical harmonic degree $(\ell)$ for two modes, with $k=8$ and $k=9$, which was only possible because the stellar light curve was dominated by a single mode in 1996. The independent spectra provide the following values: $T_{\text {eff }}=24100 \pm 400 \mathrm{~K}, \log g=7.91 \pm 0.26$ and $d=42.7 \pm 2.5 \mathrm{pc}$. The ultraviolet spectroscopic distance is in better agreement with the seismological value, than the one derived by parallax.
\end{abstract}

Key words. stars: white dwarfs - stars: variables: general - stars: oscillations - stars: individual: GD 358 - stars: evolution

\section{Introduction}

GD 358, also called V777 Herculis, is the prototype of the helium atmosphere pulsating white dwarf stars, the DBVs. It was the first pulsating star detected based on a theoretical prediction (Winget et al. 1982), and is the one with the largest number of detected periodicities, after the Sun. Detecting as many modes as possible is extremely important as each detected periodicity yields an independent constraint on the star's structure, and identifying the pulsation indices is crucial to allow real measurements. The study of pulsating white dwarf stars has already allowed us to measure stellar mass and composition layer thickness, to probe the physics at high densities, including crystallization, and has provided a chronometer to measure the age of the oldest stars and consequently, the age of the Galaxy (Winget et al. 1987; Hansen et al. 2002; Hansen \& Liebert 2003).

Beauchamp et al. (1999) studied the optical spectra of the pulsating DBs to determine their impure instability strip at $22400 \mathrm{~K} \leq T_{\text {eff }} \leq 27800 \mathrm{~K}$, and found $T_{\text {eff }}=24900 \mathrm{~K}$ $(24700 \mathrm{~K}$ from models with traces of $\mathrm{H}), \log g=7.91$ for the brightest DBV, GD $358(V=13.85)$, assuming no photospheric $\mathrm{H}$. This $\mathrm{H}$ absence was confirmed by Provencal et al. (2000), who studied the HST and EUVE spectra, deriving $T_{\text {eff }}=27000 \pm 1000 \mathrm{~K}$. They also detected Ly $\alpha$, probably from the interstellar medium.

Winget et al. (1994) reported the analysis of $154 \mathrm{~h}$ of nearly continuous time series optical photometry on GD 358, obtained during the Whole Earth Telescope (WET) ${ }^{1}$ run of May 1990. The Fourier temporal spectrum of the light curve was dominated by periodicities in the range $1000-2400 \mu \mathrm{Hz}$, with more than 180 significant peaks. They identify all of the triplet frequencies as having spherical harmonic degree $\ell=1$ and, from the details of the triplet (differing $k$ ) spacings, Bradley \& Winget (1994) derived the total stellar mass as $0.61 \pm 0.03 M_{\odot}$, the mass of the outer helium envelope as $(2.0 \pm 1.0) \times 10^{-6} M_{*}$, the luminosity as $0.050 \pm 0.012 L_{\odot}$ and, deriving a temperature and bolometric correction, the distance as $42 \pm 3 \mathrm{pc}$.

As a clear demonstration of the power of asteroseismology, Metcalfe et al. (2001, 2002), and Metcalfe (2003) used GD 358 observed periods from Winget et al. (1994) and a genetic algorithm to search for the optimum theoretical model with static diffusion envelopes, and constrained the ${ }^{12} \mathrm{C}(\alpha, \gamma){ }^{16} \mathrm{O}$ cross section, or the envelope/core symmetry (Metcalfe et al. 2003; Montgomery et al. 2003). On the other hand, Dehner \& Kawaler (1995), Brassard \& Fontaine (2003) and Fontaine \& Brassard (2002) show that a thin helium envelope is consistent with the evolutionary models starting as PG1159 models and ending as DQs, as diffusion is still ongoing around $25000 \mathrm{~K}$ and lower temperatures. Therefore, there could be two transition zones in the envelope, one between the He envelope and the $\mathrm{He} / \mathrm{C} / \mathrm{O}$ layer, where diffusion is still separating

\footnotetext{
1 The WET is a collaboration of astronomers to observe variable stars with periods of a few minutes, typical of pulsating white dwarf stars (Nather et al. 1990).
} 
the elements, and another transition between this layer and the $\mathrm{C} / \mathrm{O}$ core. The ongoing diffusion has also been calculated by Córsico et al. (2002), and the double layer structure by Althaus \& Córsico (2004).

The average IUE spectra of GD 358, consisting of 15 images taken over 11 years, from 1981 to 1992, which we will use in Sect. 3 to derive $T_{\text {eff }}, \log g$ and distance $(d)$, was published by Holberg et al. (2002).

In this paper we also report the determination in $T_{\text {eff }}, \log g$ and distance $(d)$ from our own FOS and STIS spectra in Sect. 3, and the pulsation indices for the main pulsations in Sect. 4.

\section{Observations with HST}

By observing the DBV star GD 358 with the HST, we have a unique opportunity to cross-calibrate the identification of the triplets by their period spacings (Winget et al. 1994; Kepler et al. 2003) and the amplitude variation with wavelength. The chromatic amplitude variation method was used by Robinson et al. (1995), Kepler et al. (2000), and Castanheira et al. (2004). Two stars have their modes clearly identified by the period distribution method: PG 1159-035 (Winget et al. 1991) and GD 358 (Winget et al. 1994). The stars PG 2131+066 (Kawaler et al. 1995), PG 1707+427 (Kawaler et al. 2004) and RX J2117 (Bond et al. 1996) have modes identified as $\ell=1$ from their period spacings and/or the presence of triplets.

We observed GD 358 for $4 \mathrm{~h}$ of time-resolved spectroscopy using the Faint Object Spectrograph (FOS) on the Hubble Space Telescope (HST), in August 1996 (Nitta et al. 1998). Unexpectedly, the pulsation spectra changed dramatically, passing basically to a single mode large amplitude pulsation, which we will call sforzand ${ }^{2}$, and was called "forte" by Kepler et al. (2003).

We also observed this star for $7.5 \mathrm{~h}$ with the Space Telescope Imaging Spectrograph (STIS) in May 2000, when the Fourier spectrum indicates that the star had returned to its previous normal multiperiodic pulsation state.

The exposures with FOS used the blue Digicon detector and the G160L grating, and consist of 764 useful pixels over the spectral region from 1150 to $2510 \AA$ (UV), with $1.74 \AA$ per pixel. Simultaneously to the UV spectra, we have the zeroth-order observation, with an effective wavelength at $3400 \AA$, which has a counting rate around 100 times larger than the UV data. Our FOS data has a temporal frequency resolution of $23 \mu \mathrm{Hz}$. The exposures with STIS used the G140L grating, and consist of 833 useful pixels over the spectral region 1138 to $1736 \AA$, with $0.6 \AA$ per pixel. Our STIS data has a temporal frequency resolution of $5.5 \mu \mathrm{Hz}$. The UV relative photometry from STIS and FOS quoted in Table 2 were obtained by summing the ultraviolet time-resolved spectra over all wavelengths. Simultaneous optical data to STIS was obtained with the WET.

We also detected in the HST data a periodicity of around $45 \mathrm{~min}$, which is caused by the movement of the star in the aperture caused by the wobbling of the HST solar panels when

\footnotetext{
${ }^{2}$ Sforzando is a musical term that means a sudden increase in intensity that dies away.
}

Table 1. Atmospheric parameters derived from independent UV spectra.

\begin{tabular}{cccc}
\hline \hline Instrument & $T_{\text {eff }}(\mathrm{K})$ & $\log g$ & $d(\mathrm{pc})$ \\
\hline FOS & $23900 \pm 1100$ & $7.92 \pm 0.44$ & $42.7 \pm 4.3$ \\
STIS & $24200 \pm 500$ & $7.89 \pm 0.50$ & $43.3 \pm 5.7$ \\
\hline IUE & $24000 \pm 800$ & $7.91 \pm 0.43$ & $42.3 \pm 3.8$ \\
\hline
\end{tabular}

they come in and out of the shadow of the Earth. We included this periodicity in our multisinusoidal fit, to reduce the uncertainties.

We can measure reliable pulsation amplitudes only for bins redder than approximately $1200 \AA$, because of contamination of the observed spectra by geocoronal emission.

\section{Ultraviolet spectra}

As we still have no explanation for why the pulsation feature changed so drastically in August-September 1996 and returned to the normal state after a month, as reported by Nitta et al. (1998) and Kepler et al. (2003), we started by constraining the atmospheric parameters prior to the change. We used the recalibrated average International Ultraviolet Explorer satellite (IUE) spectra taken over 11 years (from 1981 to 1992) published by Holberg et al. (2003) to derive the effective temperature, surface gravity and distance. It has a wavelength coverage from $1150 \AA$ to $3100 \AA$. We do not know the state of GD 358 when the IUE spectra were taken, but from all optical observations, since 1983, we only saw a significant change in the sforzando of August 1996.

Using a new grid of ML2 $/ \alpha=0.6$ Koester's model atmospheres, described in Finley et al. (1997), our best simultaneous fit for $T_{\mathrm{eff}}, \log g$ and $d$ is $24000 \pm 800 \mathrm{~K}, 7.91 \pm 0.43$ and $d=42.3 \pm 3.8 \mathrm{pc}$. The distance is consistent with the value derived by seismology, $42 \pm 3$ pc (Bradley \& Winget 1994), but not within one sigma of the one obtained from parallax measurement, $36 \pm 4$ pc (Harrington et al. 1985).

Fitting the three parameters for both FOS and STIS spectra, independently, we find the results shown in Table 1.

We show in Fig. 1 all the UV spectra fitted and the best model. The FOS and STIS spectra match the same model as the IUE spectra within their uncertainties, showing the star did not change its global parameters - effective temperature and radius - during the sforzando in 1996. The average values, from the three independent fits, are $T_{\text {eff }}=24100 \pm 400 \mathrm{~K}, \log g=$ $7.91 \pm 0.26$ and $d=42.7 \pm 2.5$.

We also tested for the possibility of significant extinction in the ultraviolet spectra. Thejll et al. (1991) analyzed the available IUE spectra, allowing for an extinction according to Seaton's (1979) reddening, but found none. Our IUE spectrum includes more data than the one they analyzed, and includes the final calibration of IUE (Holberg et al. 2003). As the interstellar feature around $2200 \AA$ is not detected in the spectra, extinction larger than $E_{B-V}=0.01$ is not likely. Our tests show extinction would not improve the fits, which is consistent with there being no significant extinction. 

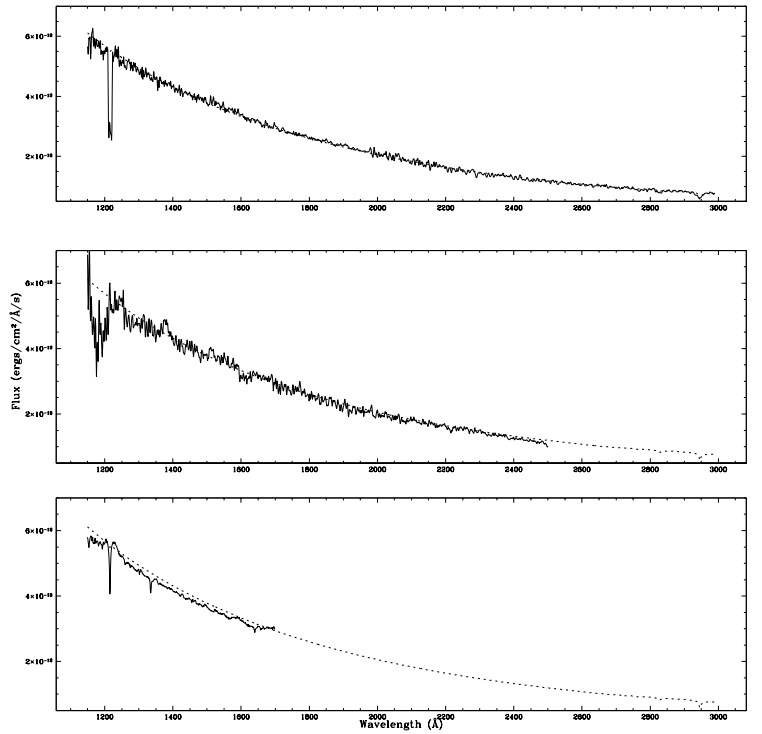

Fig. 1. Ultraviolet spectra taken with IUE (upper panel), FOS (middle panel), and STIS (lower panel), all in full line. The best model (dashed line) is at $d=42.3 \mathrm{pc}$ with $T_{\text {eff }}=24000$, and $\log g=7.91$, in all three panels.

When we fit the intrinsically high $S / N$ STIS spectra obtained in 2000, we note its shape does not agree in detail with any of our models. Considering that the highest $S / N$ spectra of the standard star G 191-B2B in the STIS archives has only $S / N \sim 20$, we believe the problem lies in the flux calibration of the spectra.

\section{Amplitude variation with wavelength}

Having determined that GD 358 did not change globally, we are ready to apply the chromatic amplitude variation method to derive atmospheric parameters in comparison to the ones derived through the spectra.

We are trying to answer the question: is the pulsation $T_{\text {eff }}$ the same as the model atmospheric $T_{\text {eff }}$, considering that the region of period formation samples deeper layers, as the $k=$ 8 mode, dominant during the sforzando, is probably trapped in the $\mathrm{C}$ to He transition layers?

To detect which periodicities are present in the data, we calculated the Fourier transform (FT) shown in Fig. 2: summed UV (right) and zeroth-order (left) light curve (top panels), prewhitening of the largest amplitude periodicity at $423.2 \mathrm{~s}$ (topmiddle panel) and only other significant periodicity at $464.2 \mathrm{~s}$ (bottom-middle panel) and the spectral window (bottom panel). The non-linear least squares fit for the two detected periodicities is listed in Table 2. During this observation, the energy transported by several modes present in previous runs was chained basically into only one mode at $423.2 \mathrm{~s}$, identified as $k=8$ by Winget et al. (1994) and Kepler et al. (2003), which had never been the largest amplitude mode in the reported data sets to date. Note that the $k=8$ mode observed in 1996 was the $m=0$ mode, not the $m= \pm 1$ modes observed in 2000. Kepler et al. (2003) indicate that after one month, the pulsation spectra returned to the multiperiodic state.
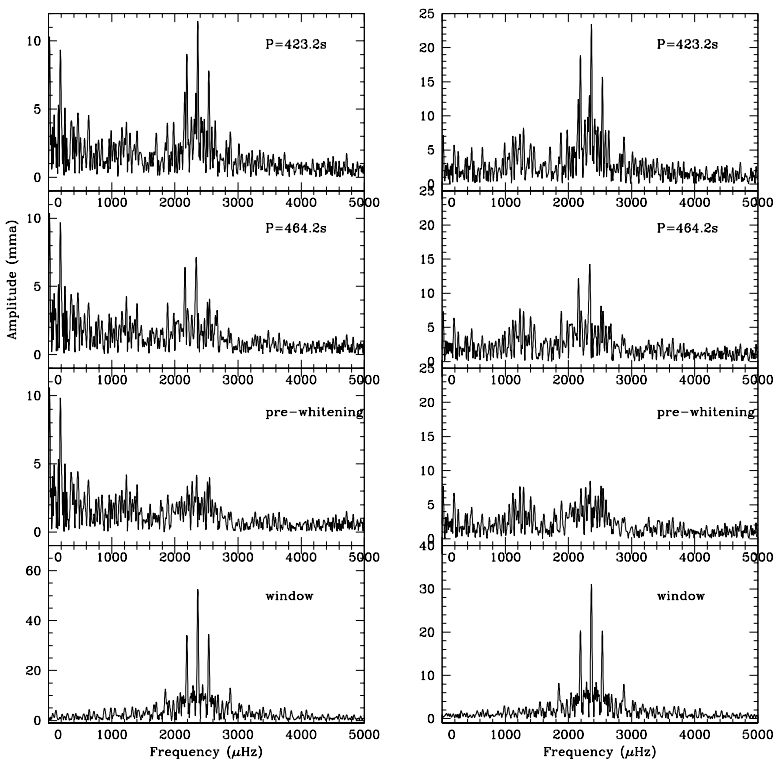

Fig. 2. The Fourier transform of the FOS data, obtained in 1996, for summed UV (right panels) and zeroth-order data (left panel). From top to bottom, we show the dominant mode around $P=423 \mathrm{~s}(k=8$, $m=0)$, another periodicity at $464.2 \mathrm{~s}(k=9, m=0)$ identified by prewhitening analysis, the noise after both subtraction, and the spectral window. The mode identification is described by Kepler et al. (2003).

Robinson et al. (1982) and Kepler (1984) demonstrated that the variable white dwarf stars pulsate in non-radial g-modes, with buoyancy providing the restoring force. They also show that the amplitudes change with wavelength but the phases do not, in the models with no non-adiabatic effects.

The chromatic amplitude variation method was applied to these two modes detected in this data set. To obtain significant amplitudes directly comparable to normalized binned model values, we convolved the time-resolved FOS spectra into bins of $50 \AA$. Then, we run a multisinusoidal nonlinear least squares fit for each convolved spectrum and for the zeroth-order (at $3400 \AA$ ), using periodicities fixed in frequency. We then compared the observational changes in amplitude with wavelength to those values predicted by the $g$-mode pulsation models described by Robinson et al. (1995) and Kepler et al. (2000), for the whole grid of $T_{\text {eff }}$ and $\log g$ models. In Fig. 3, we show how the amplitude, normalized at $3400 \AA$, changes with wavelength for the modes at $P=423.2 \mathrm{~s}$ and at $P=464.2 \mathrm{~s}$, in comparison with the theoretical ones calculated from Koester's model atmospheres with $T_{\text {eff }}=24000 \mathrm{~K}$ and $\log g=8.0$, for $\ell=1$ and $\ell=2$. Kepler et al. (2000) show in their Fig. 1 the theoretical chromatic amplitudes for $\ell=1$ to 4 .

These amplitude calculations take into account the wavelength dependence of the limb darkening and different cancellation of the flux variation for different spherical harmonic degrees. Even though Ising \& Koester (2001) and Weidner \& Koester (2003) show that the effect of the convective zone introduces nonlinearities, the amplitude and inclination angle dependence in $A(\lambda)$ are negligible for small (or large) amplitudes.

At this point, we are ready to derive the $T_{\text {eff }}$ independent of any other method. By fitting $A(\lambda) / A(3400 \AA)$ to those predicted by the models, which are $\ell$ dependent, we determined $T_{\text {eff }}$ 
Table 2. Periodicities identified using pre-whitening analysis for the HST FOS light curve, during the sforzando, in 1996, both for the total UV (1196-2484 Å) and zeroth-order light obtained with HST FOS. The times of maxima $\left(T_{\max }\right)$ for UV and zeroth-order data are given in relation to $T_{0}=2450311.8058746 \mathrm{BCT}$.

\begin{tabular}{ccccc}
\hline \hline $\begin{array}{c}\text { Period } \\
(\mathrm{s})\end{array}$ & $\begin{array}{c}\text { Amplitude (UV) } \\
(\mathrm{mma})\end{array}$ & $\begin{array}{c}T_{\max } \\
(\mathrm{s})\end{array}$ & $\begin{array}{c}\text { Amplitude (3400 ̊) } \\
(\mathrm{mma})\end{array}$ & $\begin{array}{c}T_{\max } \\
(\mathrm{s})\end{array}$ \\
\hline 464.248 & $9.80 \pm 0.62$ & $132.3 \pm 4.7$ & $4.97 \pm 0.40$ & $140.7 \pm 6.0$ \\
423.200 & $22.51 \pm 0.62$ & $187.5 \pm 1.9$ & $11.01 \pm 0.40$ & $191.0 \pm 2.5$ \\
\hline
\end{tabular}

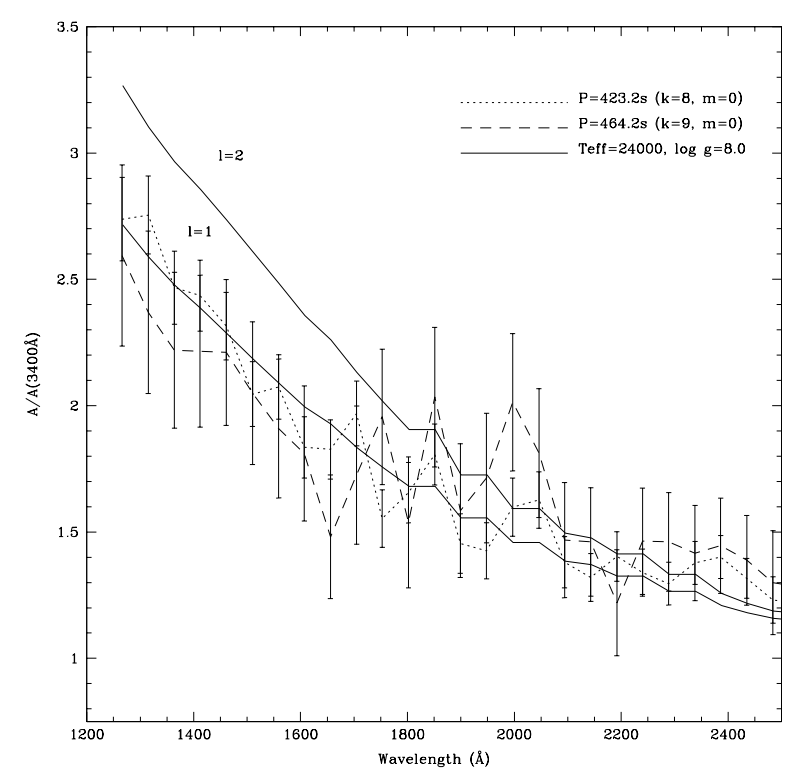

Fig. 3. Chromatic amplitude changes the way the amplitudes normalized at $3400 \AA$ changes with wavelength. The main mode at $P=423.2 \mathrm{~s}$ and the mode at $P=464.2 \mathrm{~s}$ are most probably $\ell=1$.

and $\ell$, using only the values for $\log g$ derived from the spectra, for each periodicity. Kepler et al. (2000) determined $\ell$ for the main periodicities of the DAVs G 185-32, G 226-29 and the DBV PG $1351+489$, using a fixed $T_{\text {eff }}$ and $\log g$, calculated by other methods. Castanheira et al. (2004) determined $\ell$ for G 185-32 modes, keeping all parameters free, using the constraint that solutions for each mode must result in the same $T_{\text {eff }}$ and $\log g$.

As each periodicity may fit a different value for $T_{\text {eff }}$, we calculated the local minima in $\chi^{2}$, which are the possible solutions in the difference between the observed amplitude versus wavelength curve and the models (predicted amplitudes) for each periodicity. Using a normal distribution, we estimated probability densities of that local minimum fit. Because we do not know the $\ell$ values for each periodicity, their probability must be added, i.e., the probability for each $\left(T_{\text {eff }}\right)$ model is the sum of the probabilities for $\ell=1$ to 4 . Higher values of $\ell$ were discarded because of the extremely high geometrical cancellation in the optical (Robinson et al. 1982). For each periodicity, we summed all probability densities resulting from local minima. By multiplying all the sums for the different periodicities, we obtain the most probable value of $T_{\text {eff }}=23400_{-1800}^{+3000} \mathrm{~K}$, in excellent agreement with the one derived from the spectra. The $T_{\text {eff }}$ solution is independent of the $\log g$ for this chromatic amplitude analysis. The probability

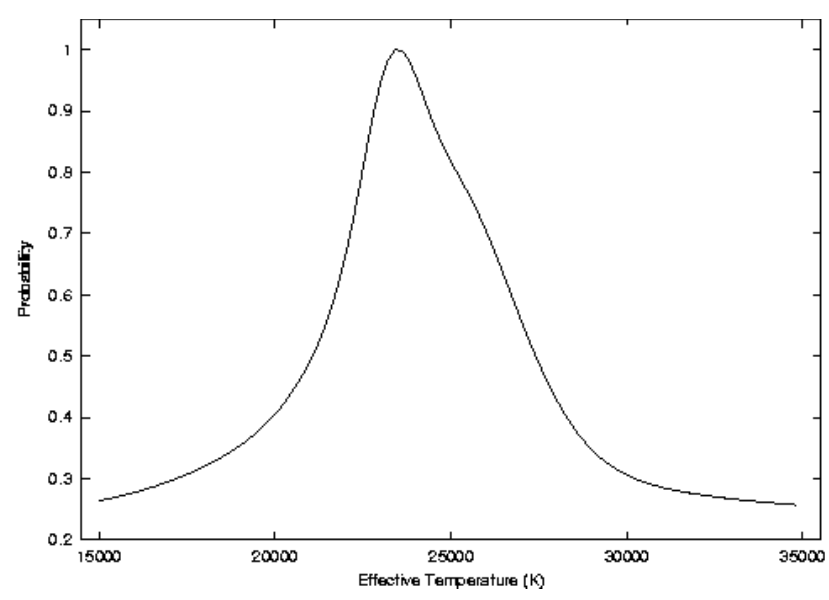

Fig. 4. The probability distribution of effective temperature derived by amplitude variation with wavelength, using the two periodicities detected with FOS data in 1996. The most probable value is $23400_{-1800}^{+3000} \mathrm{~K}$.

Table 3. Probability $(P)$ for a mode to be $\ell=1$ or 2 , for the best model, with $T_{\text {eff }}=23900 \mathrm{~K}$ and $\log g=7.91$, in the FOS data.

\begin{tabular}{ccc}
\hline \hline Period $(\mathrm{s})$ & $P(\ell=1)$ & $P(\ell=2)$ \\
\hline 423.2 & $85 \%$ & $15 \%$ \\
464.2 & $72 \%$ & $28 \%$ \\
\hline
\end{tabular}

distribution for $\log g=7.9$ models, derived by amplitude vs. wavelength (or $A(\lambda) / A(3400 \AA)$ variation) is shown in Fig. 4 . For the most probable fit, the best $\ell$ values for each mode are $\ell=1$, or 2 but with less probability, as shown in Table 3 . This is the first accurate determination of $\ell$ for a DBV using the chromatic amplitudes of pulsations. We demonstrated that the chromatic amplitude variation method works and can provide mode identifications.

To test if non-adiabatic effects, which could have arrived with the sforzando, were present we calculated the difference between the times of the maxima of the pulsation at different wavelengths. In Fig. 5 we show that phases for both $P=423.2 \mathrm{~s}$ and $P=464.2 \mathrm{~s}$ pulsations do not change with wavelength, which agrees with theoretical predictions, if non-adiabatic effects are not important (Robinson et al. 1982). This is not the case for the $142 \mathrm{~s}$ pulsation of the DAV G 185-32 as demonstrated by Thompson et al. (2004).

\section{Concluding remarks}

In spite of the remarkable change in pulsation properties, the physical parameters of the star did not change 


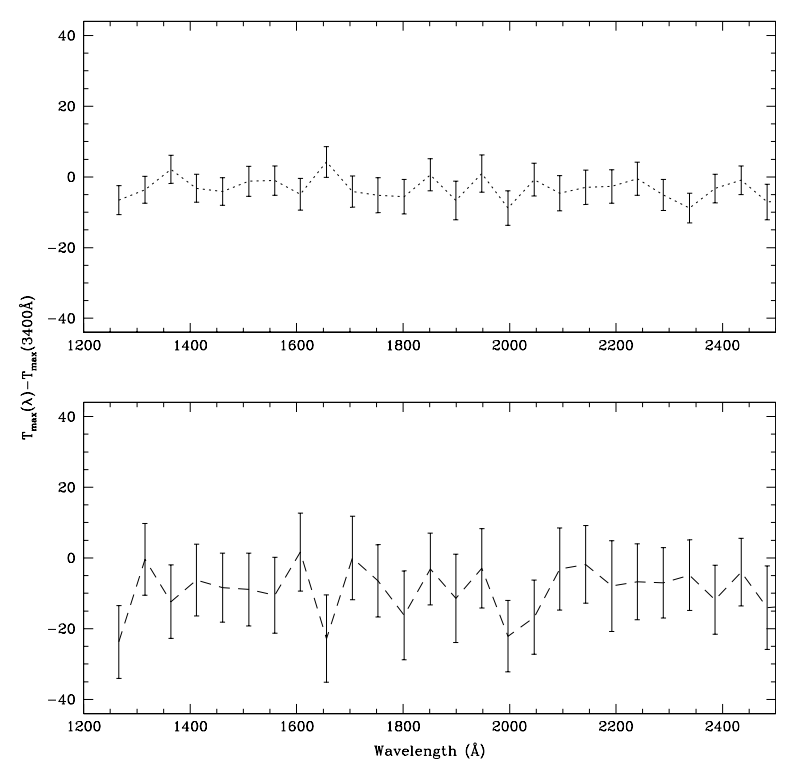

Fig. 5. The phases (times of maxima) do not change with wavelength for detected periodicities at $P=423.2 \mathrm{~s}$ (upper panel) and at $P=464.2 \mathrm{~s}$ (lower panel), in FOS data, in accordance with an $\ell=1$ mode.

when the pulsations changed in 1996. From the UV spectra we derived the distance $d=42.7 \pm 2.5 \mathrm{pc}$, effective temperature of $T_{\mathrm{eff}}=24100 \pm 400 \mathrm{~K}$ and surface gravity of $\log g=7.91 \pm 0.26$.

When the star changed basically to one dominant mode, in 1996, the amplitude variation with wavelength provides an effective temperature in agreement with the value derived from the spectra, i.e., the independent determination of the physical quantities of pulsating white dwarf stars agrees with the value derived by ultraviolet spectra when the star has few modes or the data is long enough to resolve all pulsations. We could derive $\ell=1$ for the $k=8$ and $k=9$ modes from the 1996 data because these two pulsations were resolved even in the short HST data.

These results show that we can apply the chromatic amplitude method for pulsating white dwarf stars with few modes or acquire long data sets with HST, allowing us to measure their internal structure, a consequence of their prior evolution.

Acknowledgements. Financial support: NASA-HST grant, CAPES/ UT grant, CNPq fellowship.

\section{References}

Althaus, L. G., \& Córsico, A. H. 2004, A\&A, 417, 1115

Beauchamp, A., Wesemael, F., Bergeron, P., et al. 1999, ApJ, 516, 887
Bond, H. E., Kawaler, S. D., Ciardullo, R., et al. 1996, AJ, 112, 2699

Bradley, P. A., \& Winget, D. E. 1994, ApJ, 430, 850

Brassard, P., \& Fontaine, G. 2003, NATO ASIB Proc., White Dwarfs, 105,259

Castanheira, B. G., Kepler, S. O., Moskalik, P., et al. 2004, A\&A, 413, 623

Dehner, B. T., \& Kawaler, S. D. 1995, ApJ, 445, L141

Finley, D. S., Koester, D., \& Basri, G. 1997, ApJ, 488, 375

Fontaine, G., \& Brassard, P. 2002, ApJ, 581, L33

Hansen, B. M. S., Brewer, J., Fahlman, G. G., et al. 2002, ApJ, 574, L155

Hansen, B. M. S., \& Liebert, J. 2003, ARA\&A, 41, 465

Harrington, R. S., Kallarakal, V. V., Christy, J. W., et al. 1985, AJ, 90, 123

Holberg, J. B., Barstow, M. A., \& Burleigh, M. R. 2003, ApJS, 147, 145

Ising, J., \& Koester, D. 2001, A\&A, 374, 116

Kawaler, S. D., O’Brien, M. S., Clemens, J. C., et al. 1995, ApJ, 450, 350

Kawaler, S. D., Sekii, T., \& Gough, D. 1999, ApJ, 516, 349

Kawaler, S. D., Potter, E. M., Vuckovic, M., et al. 2004, A\&A, 428, 969

Kepler, S. O. 1984, ApJ, 286, 314

Kepler, S. O., Robinson, E. L., Koester, D., et al. 2000, ApJ, 539, 379

Kepler, S. O., Nather, R. E., Winget, D. E., et al. 2003, A\&A, 401, 639

Metcalfe, T. S., Winget, D. E., \& Charbonneau, P. 2001, ApJ, 557, 1021

Metcalfe, T. S., Salaris, M., \& Winget, D. E. 2002, ApJ, 573, 803

Metcalfe, T. S. 2003, ApJ, 587, L43

Metcalfe, T. S., Montgomery, M. H., \& Kawaler, S. D. 2003, MNRAS, 344, L88

Montgomery, M. H., Metcalfe, T. S., \& Winget, D. E. 2003, MNRAS, 344,657

Musielak, Z. E., Noble, M., Porter, J. G., \& Winget, D. E. 2003, ApJ, 593, 481

Nather, R. E., Winget, D. E., Clemens, J. C., Hansen, C. J., \& Hine, B. P. 1990, ApJ, 361, 309

Nitta, A., Kepler, S. O., Winget, D. E., et al. 1998, Baltic Astron., 7, 203

Provencal, J. L., Shipman, H. L., Thejll, P., \& Vennes, S. 2000, ApJ, 542,1041

Robinson, E. L., Kepler, S. O., \& Nather, R. E. 1982, ApJ, 259, 219

Robinson, E. L., Mailloux, T. M., Zhang, E., et al. 1995, ApJ, 438, 908

Seaton, M. J. 1979, MNRAS, 187, 73

Thejll, P., Vennes, S., \& Shipman, H. L. 1991, ApJ, 370, 355

Thompson, S. E., Clemens, J. C., van Kerkwijk, M. H., O'Brien, M. S., \& Koester, D. 2004, [arXiv: astro-ph/0404577]

Weidner, C., \& Koester, D. 2003, A\&A, 406, 657

Winget, D. E., Robinson, E. L., Nather, R. D., \& Fontaine, G. 1982, ApJ, 262, L11

Winget, D. E., Hansen, C. J., Liebert, J., et al. 1987, ApJ, 315, L77

Winget, D. E., Nather, R. E., Clemens, J. C., et al. 1994, ApJ, 430, 839 